\title{
Acquired irinotecan resistance is accompanied by stable modifications of cell cycle dynamics independent of MSI status
}

\author{
AMÉLIE PETITPREZ ${ }^{1-4}$, VIRGINIE POINDESSOUS ${ }^{1-3}$, DJAMILA OUARET ${ }^{1-3}$, MARIE REGAIRAZ $^{5}$, \\ GÉRARD BASTIAN $^{3,6}$, ERIC GUÉRIN ${ }^{7}$, ALEXANDRE E. ESCARGUEIL ${ }^{1-3}$ and ANNETTE K. LARSEN ${ }^{1-3}$ \\ ${ }^{1}$ Laboratory of Cancer Biology and Therapeutics, Saint-Antoine Research Center; ${ }^{2}$ National Institute \\ for Health and Medical Research (INSERM), U938; ${ }^{3}$ Pierre and Marie Curie University (UPMC); \\ ${ }^{4}$ Paris Descartes University, Paris; ${ }^{5}$ Ecole Normale Supérieure (ENS) de Cachan, Cachan; \\ ${ }^{6}$ Pitié-Salpêtrière Hospital, Paris; ${ }^{7}$ University of Strasbourg, EA 4438, Strasbourg, France
}

Received December 15, 2012; Accepted February 12, 2013

DOI: 10.3892/ijo.2013.1868

\begin{abstract}
Irinotecan is a major anticancer agent specifically targeting DNA topoisomerase I. Its cytotoxicity is mediated via a two-step process involving accumulation of reversible DNA-topoisomerase I complexes associated with transient DNA single-strand breaks which subsequently are converted into permanent DNA double-strand breaks by the replication fork during $\mathrm{S}$ phase. Irinotecan may be selectively active for treatment of colorectal cancers that show microsatellite instability (MSI) due to deficiencies in mismatch repair enzymes, compared to tumors that are microsatellite stable but show chromosome instability (CIN). Although the clinical activity of irinotecan is principally limited by acquired drug resistance, surprisingly little is known about the influence of prolonged irinotecan exposure on the cell cycle dynamics. We have developed two colon cancer cell lines resistant to $\mathrm{SN}-38$, the active metabolite of irinotecan, one derived from HT-29 (CIN), the other from HCT-116 (MSI). We here show that besides classical resistance mechanisms, $\mathrm{SN}-38$ resistance is accompanied by an increased generation doubling time, a decreased $\mathrm{S}$ phase fraction and an increased G2 fraction in vitro as in tumor xenografts for both CIN and MSI models. As a consequence, SN-38-resistant cells and tumors show cross-resistance to the S-phase selective agent 5-fluorouracil. The resistance is accompanied by increased basal levels of $\gamma-\mathrm{H} 2 \mathrm{AX}$ and phospho-Chk2 without notable changes in the levels of phospho-Chk1. Taken together, our results show that prolonged irinotecan exposure is accompanied by stable modifications of cell cycle dynamics which could have profound impact on tumor sensitivity to a
\end{abstract}

Correspondence to: Dr Annette K. Larsen, Laboratory of Cancer Biology and Therapeutics, Hôpital Saint-Antoine, Kourilsky Building 1st floor, 184 rue du Faubourg Saint Antoine, Paris 75571, Cedex 12, France

E-mail: annette.larsen@upmc.fr

Key words: irinotecan, resistance, colorectal cancer, cell cycle dynamics, microsatellite instability wide range of antitumor agents and may influence tumor progression in patients.

\section{Introduction}

Irinotecan (CPT-11) is a camptothecin derivative (CPT) selectively targeting the nuclear enzyme DNA topoisomerase I. Like other CPTs, irinotecan-mediated cytotoxicity is mediated by a two-step process. This includes drug-mediated accumulation of reversible DNA-topoisomerase I complexes (called cleavable complexes) that are associated with transient DNA single strand breaks. Subsequently, these complexes are converted into permanent DNA single and double strand breaks by the replication fork during the $S$ phase of the cell cycle (1-4). As a result, CPTs are preferentially toxic toward S phase cells (5-7).

Irinotecan is a major drug for treatment of patients with metastatic colorectal cancer (CRC) $(8,9)$ and a promising agent for other applications such as gastric cancer. However, its clinical activity is limited by both intrinsic (natural) and acquired drug resistance. Patients with CRC can be divided into two major groups according to the type of genetic instability displayed by the tumor $(10,11)$. The major group $(>80 \%$ of patients) is characterized by numerical and/or structural chromosome instability (CIN) associated with loss of heterozygosity $(\mathrm{LOH})$ and aneuploidy. The other group $(\sim 15 \%$ of patients) is characterized by microsatellite instability (MSI/ MIN). MSI is linked to dysfunction of the DNA mismatch repair (MMR) system, which is involved in the correction of base/base mismatches and insertion/deletion loops during replication (12). Interestingly, it has been reported that irinotecan is selectively active toward MMR-deficient tumor cells in vitro as in patients $(13,14)$ although this needs further confirmation.

Acquired CPT resistance has been associated with reduced drug uptake, decreased topoisomerase I expression and/or TOP1 mutations (14-21). In comparison, surprisingly little is known about the influence of prolonged CPT exposure on the cell cycle machinery. This issue is likely to gain importance in the years to come considering the advent of novel liposomal and pegylated preparations of irinotecan and its major metabolite SN-38 $(22,23)$. 
We have developed two independent CPT-resistant human CRC cell lines, one derived from HT-29 cells (CIN) the other from HCT-116 cells (MSI) by prolonged exposure to increasing concentrations of SN-38 in the growth media. Here, we report that in addition to classical resistance mechanisms, CPT resistance is accompanied by increased generation doubling time, a decreased $\mathrm{S}$ fraction and an increased $\mathrm{G} 2$ fraction in vitro as in vivo. As a consequence, $\mathrm{SN}-38$-resistant cells show crossresistance to $\mathrm{S}$-phase selective agents such as 5-fluorouracil. SN-38 resistance is accompanied by increased basal levels of $\gamma-\mathrm{H} 2 \mathrm{AX}$ and phospho-Chk2 without notable changes in the levels of phospho-Chk1. Taken together, our results indicate that prolonged exposure to CPTs is accompanied by stable modifications of cell cycle dynamics. This could have profound impact on tumor sensitivity to a wide range of antitumor agents and may influence tumor progression in patients.

\section{Materials and methods}

Drugs and chemicals. Irinotecan was purchased from Pfizer whereas SN-38 (7-Ethyl-10-Hydroxy-20(S)-Camptothecin) was obtained from Abatra Technology (Sophia Ho, China). 5-Fluorouracil was purchased from Teva-Pharma. MTT (3-[4,5-dimethylthiazol-2-yl])-2,5-diphenyltetrazolium bromide), propidium iodide, 4',6-diamidino-2-phenylindole (DAPI) and RNase A were obtained from Sigma.

Cells and culture medium. HCT-116 and HT-29 colorectal carcinoma (CRC) cells were generously provided by Bert Vogelstein (Baltimore, MD) and Richard Camalier (Division of Cancer Treatment and Diagnosis Tumor Repository, NCI), respectively. The cells were maintained in Mc Coy's 5A (HCT-116) or DMEM medium (HT-29) from PAA supplemented with $5 \%$ fetal calf serum (FCS, Eurobio), $100 \mathrm{U} / \mathrm{ml}$ penicillin and $100 \mu \mathrm{g} / \mathrm{ml}$ streptomycin.

To obtain SN-38-resistant cells, HT-29 and HCT-116 cells were exposed to increasing concentrations of SN-38 over 6-9 months as previously described (25). Specifically, cells in $\log$ phase were first exposed to the $\mathrm{IC}_{50}$ dose of SN-38. Once surviving cells reached $80 \%$ confluence, they were passaged twice per week at the same concentration of SN-38. The process was repeated with increasing doses of SN-38 until a resistant cell population was obtained. Cells were routinely maintained in drug-free media except for a single three day drug exposure every three weeks. All experiments were carried out with cells grown without drug for at least one week. The resistant phenotype was stable over a least 20 passages in the absence of drug.

Cytotoxicity assay. The MTT (tetrazolium dye [3-(4,5-dimethylthiazol-2-yl)-2,5-diphenyltetrazolium bromide], Sigma) assay was used to determine the growth inhibitory effects of SN-38 after $120 \mathrm{~h}$ continuous drug exposure as previously described (26-28). The $\mathrm{IC}_{50}$ value corresponds to the drug concentration inhibiting cell growth by $50 \%$ compared with the growth of untreated control cells. All values are averages of at least three independent experiments performed in duplicate.

Clonogenic assay. The colony formation assay was carried out as previously described (29). Briefly, cells (500-2,000) were plated in 60-mm Petri dishes and incubated overnight.
Exponentially growing cells were exposed to different drug concentrations for ten days and the colonies were fixed with ethanol, stained with $0.5 \%$ crystal violet and examined under a stereomicroscope. Colonies of 50 or more cells were considered to originate from viable cells. The $\mathrm{IC}_{50}$ value corresponds to the drug concentration inhibiting colony formation by $50 \%$ compared to the number of colonies for untreated control cells. All values are averages of at least three independent experiments, each performed in duplicate.

Single cell gel electrophoresis (comet assay). Cells were exposed to the indicated drug concentrations for $20 \mathrm{~min}$ at $37^{\circ} \mathrm{C}$ in the dark and subjected to single cell electrophoresis (comet assay) under alkaline conditions as described previously (30,31). Image analysis was performed using Komet 5.5 software (Kinetic Imaging, Nottingham, UK) to determine the percentage of nuclear DNA present in the comet tail. A minimum of 100 cells were analyzed per sample. Values represent the average of at least two independent experiments.

Relative quantitative RT-PCR. mRNA expression of TOPI was evaluated by relative quantitative real-time PCR (qRT-PCR) as previously described $(32,33)$. Validated QuantiTect ${ }^{\circledR}$ Primer Assays (Qiagen) were used for amplification. All quantifications were done in duplicate for three independent experiments and normalized with respect to the endogenous $\beta$-actin (ACTB) mRNA levels for each reaction. Target cDNA expression was quantified using the comparative $\mathrm{C}_{\mathrm{t}}$ method and expressed as the fold change in samples from $\mathrm{SN}-38$-resistant cells vs samples from the corresponding parental cell lines.

TOP1 sequencing. Total RNA was extracted using RNeasy Plus mini kit (Qiagen) and reverse-transcribed using the RevertAid Premium reverse transcriptase (Fermentas). The resulting cDNA was used to amplify the entire TOPI open reading frame by polymerase chain reaction (PCR) and PCR products were sequenced bidirectionally (Eurofins MWG Operon, Ebersberg, Germany).

Drug uptake. HPLC was performed as previously described (34) with minor modifications. For each cell line, three independent samples of five millions cells were incubated with $150 \mathrm{nM}$ SN-38 for $20 \mathrm{~min}$ at $37^{\circ} \mathrm{C}$, rapidly washed in ice-cold PBS and scraped in $2 \mathrm{ml}$ of ice-cold methanol. Samples were centrifuged at $4^{\circ} \mathrm{C}$ (800 $\mathrm{g}$ for $\left.7 \mathrm{~min}\right)$, the cell pellets were suspended in $1 \mathrm{ml}$ methanol and samples (50 $\mu \mathrm{l}$ each) subjected to HPLC analysis.

The chromatographic detection was achieved by using an Atlantis-C18 (5 $\mu \mathrm{m}, 250 \times 4.6 \mathrm{~mm})$ analytical column (Waters) maintained at room temperature and protected by a $\mathrm{C} 18$ guard cartridge (Waters). The mobile phase was a mixture of $75 \% 20 \mathrm{mM}$ ammonium acetate, $\mathrm{pH} 3.4$ and $25 \%$ acetonitrile with a flow rate of $1.5 \mathrm{ml} / \mathrm{min}$. The fluorescence detector excitation wavelength was $368 \mathrm{~nm}$ and the emission wavelength $515 \mathrm{~nm}$. Run time for each analysis was $13 \mathrm{~min}$. The retention time for SN-38 was $9.8 \mathrm{~min}$ and the detection limit was $0.5 \mathrm{ng} / \mathrm{ml}$. Data collection and processing were performed using Millenium software (Waters). Each sample was analyzed at least twice.

Western blot analysis. Western blot analysis was carried out as described previously $(35,36)$. The following primary antibodies were used: rabbit anti-ABCG2 (\#4477), rabbit anti-pSer317 Chk1 
(\#2344), rabbit anti-pThr68 Chk2 (\#2661), mouse anti-Chk1 (\#2345) and mouse anti-Chk2 (\#2662), all from Cell Signaling. Rabbit anti-Topo I (sc-10783) was from Santa Cruz, mouse antiPgp (\#517312) was from Calbiochem, while mouse anti- $\beta$-actin (\#A5441) was purchased from Sigma. The secondary antibodies include horseradish peroxidase-conjugated goat anti-mouse or anti-rabbit secondary antibodies (Jackson ImmunoResearch Laboratories) while the enhanced chemiluminescence reagent was obtained from Amersham.

Flow cytometry analysis. The cell cycle distribution was measured by flow cytometry using a FACSCalibur flow cytometer (Becton-Dickinson) as previously described $(37,38)$. The expression of phosphorylated histones was determined with help of the following antibodies: mouse anti-pSer139 histone H2AX antibody (\#05-636, Millipore) and mouse anti-pSer10 histone H3 (Cell Signaling, 9706).

Immunocytochemistry and image acquisition. Cells were grown on coverslips and prepared for immunocytochemistry as described previously $(39,40)$. Epitopes were detected with the following primary antibodies: mouse anti-pSer139 histone H2AX, rabbit anti-Cyclin B1 (\#4138, Cell Signaling) or mouse anti-pSer1981 ATM (\#4526, Cell Signaling) followed by anti-rabbit or anti-mouse CyTM3-conjugated secondary antibodies from Jackson ImmunoResearch Laboratories. Cells were washed, counterstained with DAPI and mounted with Vectashield (Vector Laboratories) before being subjected to microscopy. Fluorescent images were captured using an inverted microscope (Olympus CKX41) and digital compact camera (Olympus Camedia C4000) and the fluorescence intensities were determined using the MetaMorph software (Universal Imaging Corporation) for quantitative analysis.

Immunohistochemistry. To measure in vivo DNA synthesis, the thymidine analog 5-ethynyl-2'-deoxyuridine (EdU, Life Technologies) was administered $48 \mathrm{~h}$ before sacrifice as previously described (41). Incorporated EdU was revealed by a fluorescent-azide coupling reaction (Click-iT, Life Technologies) of paraffin-embedded tumor samples and counterstained by DAPI to reveal the nuclei of individual cells. For quantitative analysis of in vivo DNA synthesis (EdU incorporation), the data represent the ratios between EdU-positive cells and the total number of viable cells and are the averages of five fields/tumor (each field representing approximately 1,700 cells) from three different tumors. All images were captured with a fluorescence microscope, and the fluorescence intensities were determined by the MetaMorph software for quantitative analysis.

Xenograft models. The antitumor activity of irinotecan was evaluated in athymic mice (female NMRI-Foxn1, 6 weeks old) from Taconic (Skensved, Denmark) bearing HT-29, HT-29/SN-38, HCT-116 or HCT-116/SN-38 CRC xenografts as described previously (41). Briefly, two to six million cells were injected into the right flank and the treatments were started when the tumors were palpable (median tumor volume $\sim 100 \mathrm{~mm}^{3}$ ). Animals were weighed daily and tumor sizes were determined three times per week. Measurements (in millimeter) were made in two dimensions (width and length) and tumor volumes were calculated as $\mathrm{AxB}^{2} / 2$, where $\mathrm{A}$ is tumor length and $\mathrm{B}$ is tumor width as described previously (41).

For determination of drug sensitivity, mice were treated with $35 \mathrm{mg} / \mathrm{kg}$ irinotecan i.p. every four day or with $25 \mathrm{mg} / \mathrm{kg}$ 5-FU i.p. on days 1, 2, 15 and 16 and the tumor growth inhibition was determined by comparison with the tumor growth of untreated controls.

Statistical analysis. The statistical analysis of experimental data was performed using a Student's paired t-test, and results are presented as mean \pm standard deviation (SD).

\section{Results}

Establishment of SN-38-resistant cell lines. SN-38-resistant cells were established by continuous exposure to increasing concentrations of SN-38 in the growth media for 6-9 months as previously described (25). The cytotoxicity of SN-38 toward parental and resistant CRC cells was determined by the MTT viability assay after $120 \mathrm{~h}$ continued drug exposure. The parental HCT-116 cells (MSI) were more sensitive than the parental HT-29 cells (CIN) with $\mathrm{IC}_{50}$ values of 5 and $12 \mathrm{nM}$, respectively. In contrast, the resistance to SN-38 was more pronounced for HCT-116/SN-38 cells than for HT-29/SN-38 cells with $\mathrm{IC}_{50}$ values of $90 \mathrm{nM}$ (18-fold resistance) and $60 \mathrm{nM}$ (5-fold resistance), respectively.

The formation of drug-induced single-stranded DNA breaks is reduced in SN-38-resistant cells. The CPT-induced cleavable complexes are associated with the formation of transient DNA single-strand breaks and can thus be determined by the alkaline comet assay (single cell electrophoresis) after brief drug exposure (42). The results (Fig. 1A) show that the development of SN-38 resistance was accompanied by a significant reduction in the levels of DNA single stranded breaks (that is cleavable complexes) at all doses examined for both HT-29/SN-38 and HCT-116/SN-38 cells, compared with the respective parental cells $(\mathrm{p}<0.001)$.

Expression of topoisomerase I in parental and SN-38-resistant cells. The attenuation of drug-induced DNA single-strand breaks can result from decreased levels of topoisomerase I. qRT-PCR analysis showed that the mRNA levels of topoisomerase I were significantly decreased $(\mathrm{p}<0.01)$ in HT-29/SN-38 cells whereas no notable changes were observed for HCT-116/SN-38 cells (Fig. 1B, left panel). Western blot analysis of topoisomerase I indicated a modest decrease $(20 \%)$ in topoisomerase I protein levels for both resistant cells lines, compared with the respective parental cells (Fig. 1B, right panel). These findings indicate that the important differences between parental and SN-38-resistant cells in the formation of DNA single-strand breaks (Fig. 1A) can not be explained by attenuation of topoisomerase I protein levels alone.

Topoisomerase I sequencing of parental and $\mathrm{SN}-38$ resistant CRC cells. To determine whether the decreased formation of cleavable complexes could result from TOPI mutations, we sequenced the full open reading frame of TOP1 in parental and SN-38-resistant HCT-116 and HT-29 cells. No mutations were found in any of the four cell lines, demonstrating that resistance to $\mathrm{SN}-38$ is not linked to topoisomerase I mutations (data not shown). 
A
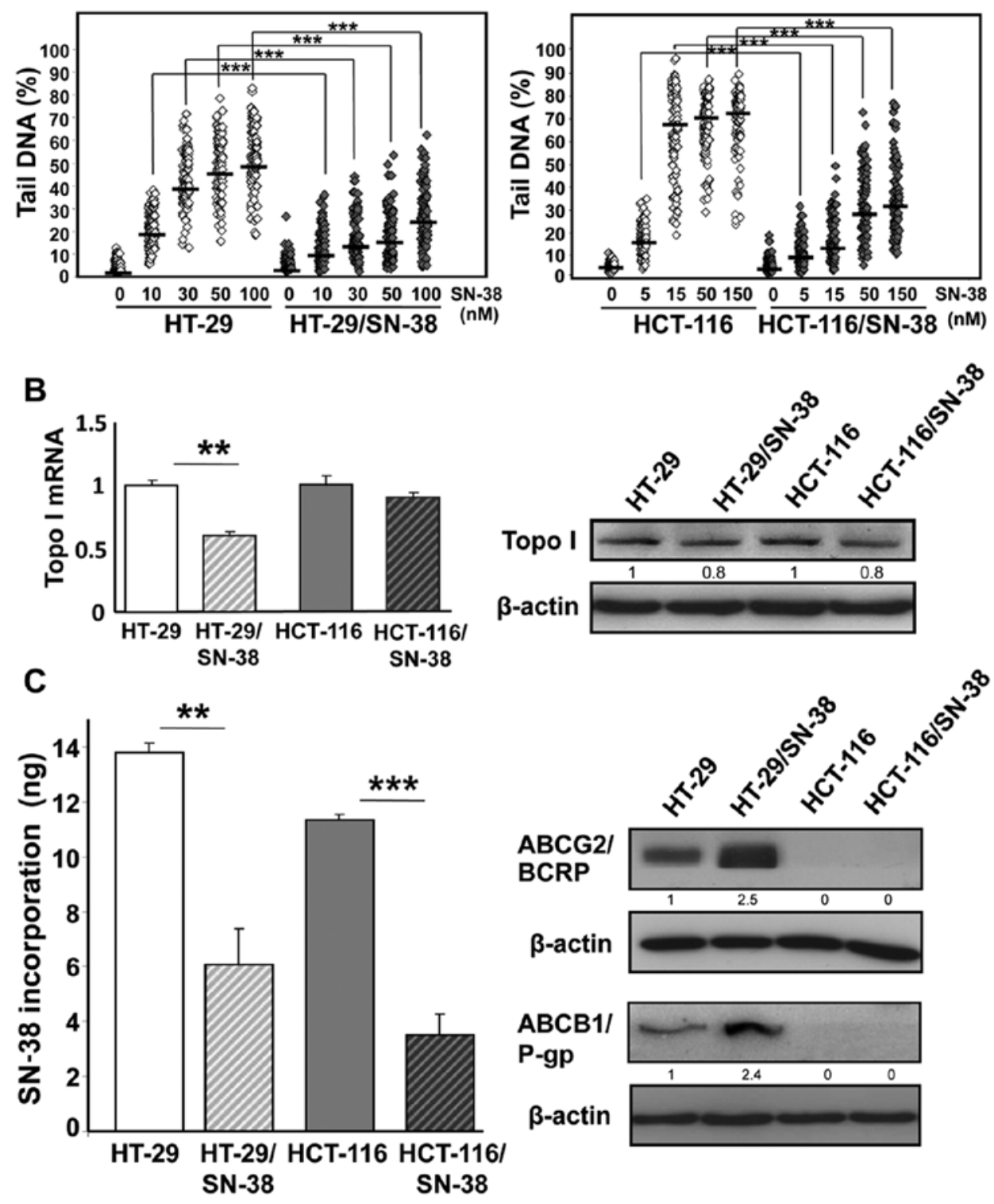

Figure 1. Molecular and functional characterization of SN-38-resistant colorectal cancer cells. (A) Influence of SN-38 on the formation of DNA single-strand breaks. Left, HT-29 $(\diamond)$ and HT-29/SN-38 $(\diamond)$ cells. Right, HCT-116 $(\diamond)$ and HCT-116/SN-38 $(\diamond)$ cells. Cells were exposed to the indicated concentrations of SN-38 for $20 \mathrm{~min}$ and the induction of DNA single strand breaks was determined by the alkaline comet assay. The results indicate the levels of DNA damage in individual cells from a typical experiment and are expressed as the \% of total DNA present in the comet tail; ${ }^{* * *}$ p $<0.001$. (B) Topoisomerase I expression in parental and SN-38-resistant colorectal cancer cells. Left, mRNA expression of topoisomerase I was determined by qRT-PCR. The columns represent the relative expression of TOP1 mRNA and are averages of three independent experiments, each carried out in duplicate and normalized with respect to the endogenous $A C T B$ mRNA levels. Bars, standard deviation; ${ }^{* *}$ p $<0.01$. Right, protein expression of topoisomerase I was determined by western blot analysis with $\beta$-actin as loading control. The numbers indicate the relative expression of topoisomerase I after standardization for equal loading. (C) Accumulation of SN-38 in parental and SN-38-resistant cells after $20 \mathrm{~min}$ exposure to $150 \mathrm{nM} \mathrm{SN-38}$ as determined by HPLC analysis. Left, the columns indicate the average values of three independent samples. Bars, standard deviation; ${ }^{* * *} \mathrm{p}<0.001 ;{ }^{* *} \mathrm{p}<0.01$. Right, expression of ABCG2 and ABCB1 was determined by western blot analysis with $\beta$-actin as loading control. The numbers indicate the relative expression of ABCG2 and ABCB1 after standardization for equal loading.

Drug accumulation is reduced in SN-38-resistant cells. Next, the accumulation of $\mathrm{SN}-38$ in parental and resistant cells was determined by HPLC analysis after brief exposure to SN-38 $(150 \mathrm{nM})$. The results (Fig. 1C, left panel) show that SN-38 resistance was accompanied by 2 - to 3 -fold reduction in SN-38 accumulation for both HT-29/SN-38 cells $(\mathrm{p}<0.01)$ and HCT-116/SN-38 cells ( $\mathrm{p}<0.001)$.

Irinotecan resistance has been associated with increased expression of drug efflux pumps belonging to the $\mathrm{ABC}$ transporter superfamily including ABCG2/BCRP and
ABCB1/p-glycoprotein $(21,22)$. In agreement, both proteins were upregulated in HT-29/SN-38 cells, compared with HT-29 parental cells. In comparison, no expression of P-glycoprotein or BCRP was detected in either parental or SN-38 resistant HCT-116 cells (Fig. 1C, right panel).

Growth and cell cycle dynamics are altered in SN-38resistant cells. Interestingly, both $\mathrm{SN}-38$-resistant cells grow slower than the corresponding parental cells with doubling times of 24 vs $30 \mathrm{~h}$ for HT-29 and HT-29/SN-38 cells, respec- 
A

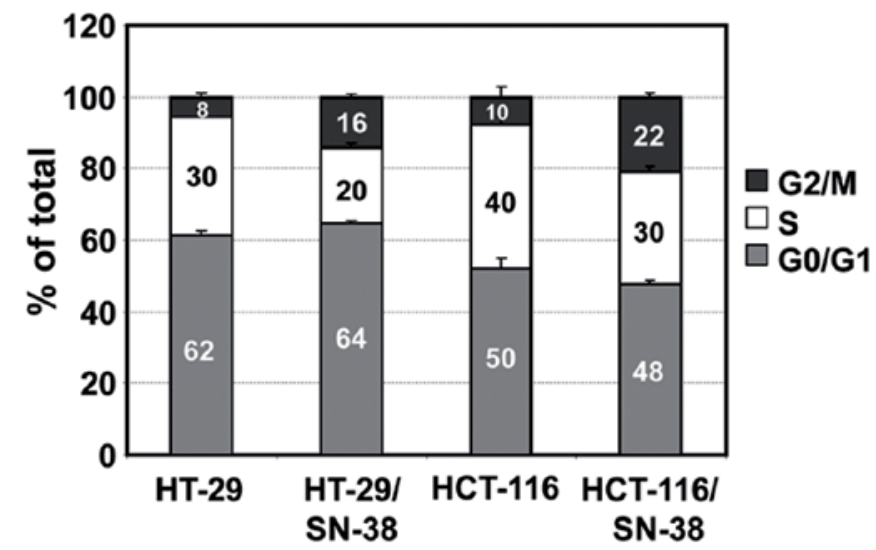

B

Cyclin B1

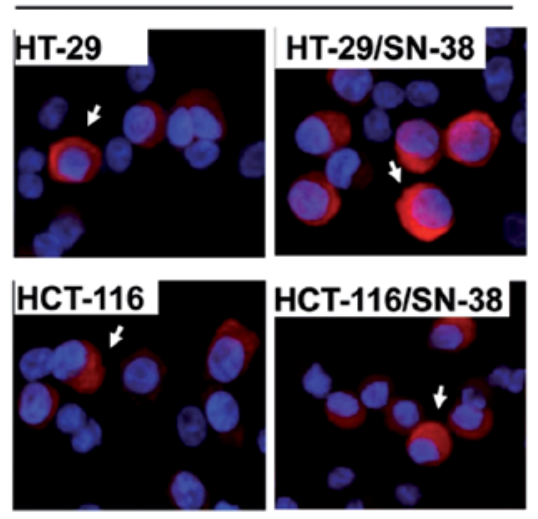

C

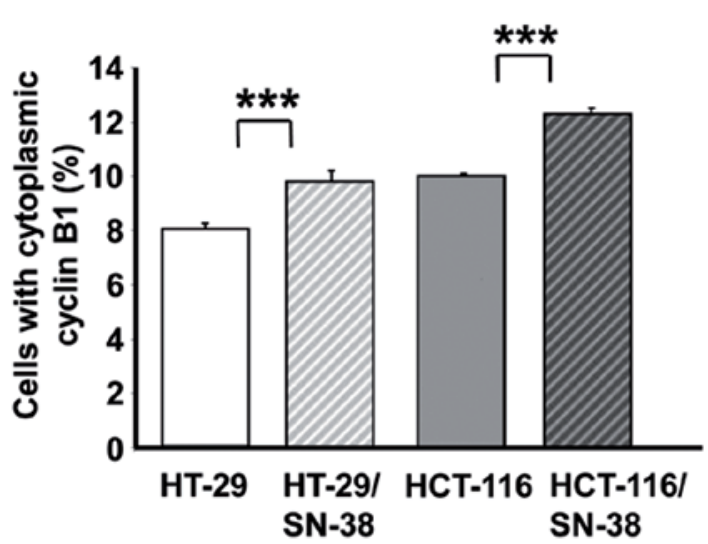

Figure 2. Cell cycle distribution of parental and SN-38-resistant colorectal cancer cells. (A) The cell cycle distribution of untreated parental and SN-38-resistant cells was determined by flow cytometry analysis. The numbers indicate the average fraction of cells in a given phase of the cell cycle as determined from at least three independent experiments. Bars, standard deviation. (B) Expression of cyclin B1 in parental and SN-38-resistant cells. The expression and cellular localization of cyclin B1 (red) was determined by immunocytochemistry. The nuclei were counterstained with DAPI (blue). (C) Quantitative analysis of cells with cytoplasmic cyclin B1. The columns represent the proportion of cells with cytoplasmic B1 from five independent experiments, each carried out with 1,000 cells. Bars, standard deviation; ${ }^{* * *} \mathrm{p}<0.001$

tively, and 22 vs $29 \mathrm{~h}$ for HCT-116 and HCT-116/SN-38 cells, respectively (data not shown). Cell cycle analysis revealed that the $\mathrm{S}$ phase fraction was reduced by one-fourth to onethird for both SN-38-resistant cell lines, whereas the G2/M fraction had at least doubled (Fig. 2A). Additional analysis for Ser10-phosphorylated histone H3, a mitotic marker (43), revealed no important differences in the fraction of phosphoH3 positive cells which varied between 3.8 and $4.5 \%$ for all four cell lines (data not shown). Therefore, the increased G2/M fraction is due to an important increase of cells in the G2 phase of the cell cycle.

For further validation, the cellular distribution of cyclin B1 was determined by immunocytochemistry. Cyclin B1 accumulates in the cytoplasm during the G2 phase and is then translocated to the nucleus during prophase before the breakdown of the nuclear membrane (44-46). Quantitative analysis of cells with cytoplasmic cyclin B1 (arrows, Fig. 2B, left panel) revealed a significant $(\mathrm{p}<0.001)$ increase in the fraction of such cells for both SN-38 resistant cell lines (Fig. 2B, right panel), in agreement with the cell cycle data.
SN-38-resistant cells show altered growth in vivo. For in vivo characterization, tumor xenografts were established from parental and SN-38-resistant cell lines. The growth of HT-29/SN-38 tumors was significantly $(\mathrm{p}<0.01)$ slower than that of HT-29 tumors with average tumor volumes of $721 \mathrm{~mm}^{3}$ vs $1,362 \mathrm{~mm}^{3}$ by 28 days, corresponding to a $47 \%$ reduction in the average tumor size (Fig. 3A). The growth of HCT-116/SN-38 tumors was also significantly different from that of HCT-116 tumors $(\mathrm{p}<0.05)$ with average tumor volumes of $1,009 \mathrm{~mm}^{3}$ for HCT-116/SN-38 compared with $1,733 \mathrm{~mm}^{3}$ for the parental HCT-116 tumors, corresponding to a $42 \%$ reduction in the average tumor size (Fig. 3B). Therefore, in both cases, tumor growth of the SN-38-resistant xenografts was approximately half of that observed for the corresponding parental tumors.

Treatment of the mice with irinotecan $(35 \mathrm{mg} / \mathrm{kg}$ i.p. every four days) revealed that the HT-29/SN-38 and HCT-116/SN-38 tumors retained the resistance to irinotecan in vivo throughout the four-week treatment. Specifically, irinotecan treatment reduced the average tumor size by $75 \%$ for HT-29 tumors 
A
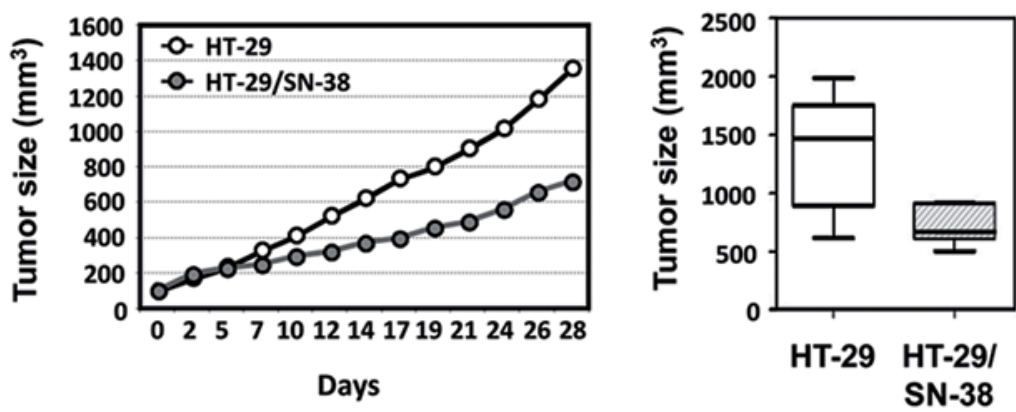

B
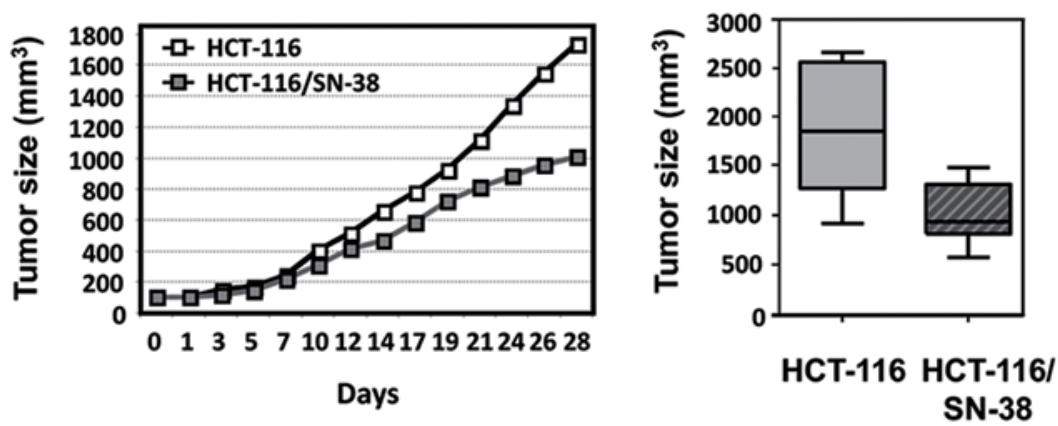

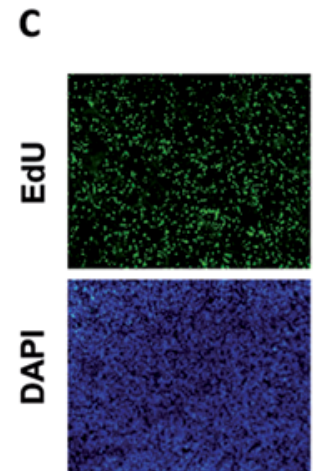

HT-29
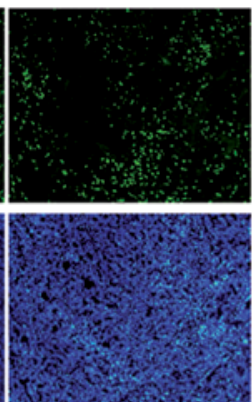

HT-29/SN-38
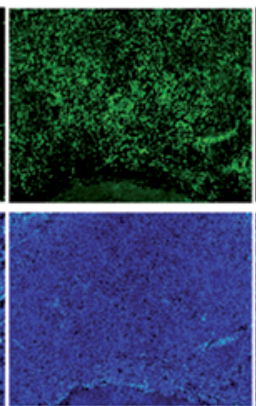

HCT-116
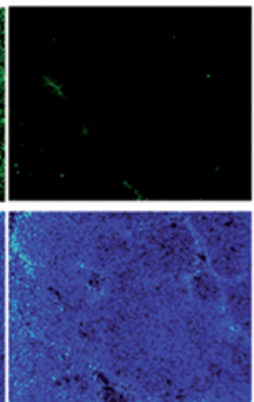

HCT-116/SN-38

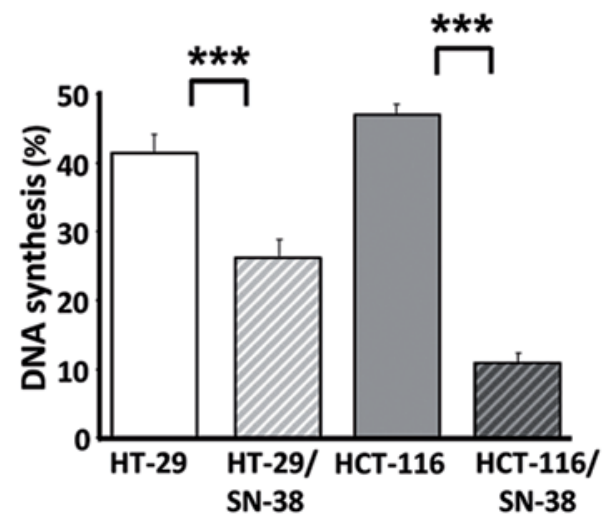

Figure 3. Growth and DNA synthesis of tumor xenografts from parental and SN-38-resistant colorectal cancer cells in nude mice. (A) Left, tumor growth of HT-29 (O) and HT-29/SN-38 (•) tumors. The curves represent the average tumor growth of at least seven animals per group. Right, box and whisker plot of tumor volumes in mice with HT-29 or HT-29/SN-38 xenografts by day 28 . Lines, medians; boxes, 25 th to 75 th percentile interquartile ranges; whiskers, the highest and lowest value for the group. (B) Left, tumor growth of HCT-116 () and HCT-116/SN-38 (- tumors. The curves represent the average tumor growth of at least 7 animals per group. Right, box and whisker plot of tumor volumes in mice with HCT-116 or HCT-116/SN-38 xenografts by day 28. Lines, medians; boxes, 25th to 75th percentile interquartile ranges; whiskers, the highest and lowest value for the group. (C) In vivo DNA synthesis of parental and SN-38-resistant tumor xenografts as measured by EdU incorporation. Left, representative images of tumors from HT-29, HT-29/SN-38, HCT-116 and HCT-116/SN-38 xenografts. Tumor cell nuclei (DAPI) appear in white in the lower panels, whereas nuclei with active DNA synthesis (EdU) appear in white in the upper panels. Right, the columns represent the ratio between EdU-positive cells and the total number of viable cells and are the averages of five fields/tumor (each field representing approximately 1,700 cells) from three different tumors. ${ }^{* * *} \mathrm{p}<0.001$.

compared with 55\% for HT-29/SN-38 tumors. Irinotecan was highly efficient toward HCT-116 tumors with complete tumor regression in one-third of the animals and approximately $95 \%$ reduction of the tumor size for the remaining tumors. In comparison, irinotecan only reduced the tumor size of HCT-116/SN-38 tumors by $37 \%$ (data not shown).

SN-38-resistant cells show reduced levels of DNA synthesis in vivo. Next, we wished to compare the levels of in vivo
DNA synthesis in parental and SN-38-resistant cells. For this purpose, tumor-bearing mice were injected with EdU, a thymidine analog, $48 \mathrm{~h}$ before sacrifice and EdU incorporation was subsequently determined by fluorescence histochemistry (Fig. 3C, left panel). Quantitative image analysis showed that DNA incorporation was significantly $(\mathrm{p}<0.001)$ lower in both SN-38-resistant tumors compared with tumors derived from the respective parental cells, which was particularly striking for the HCT-116 tumors (Fig. 3C, right panel). 

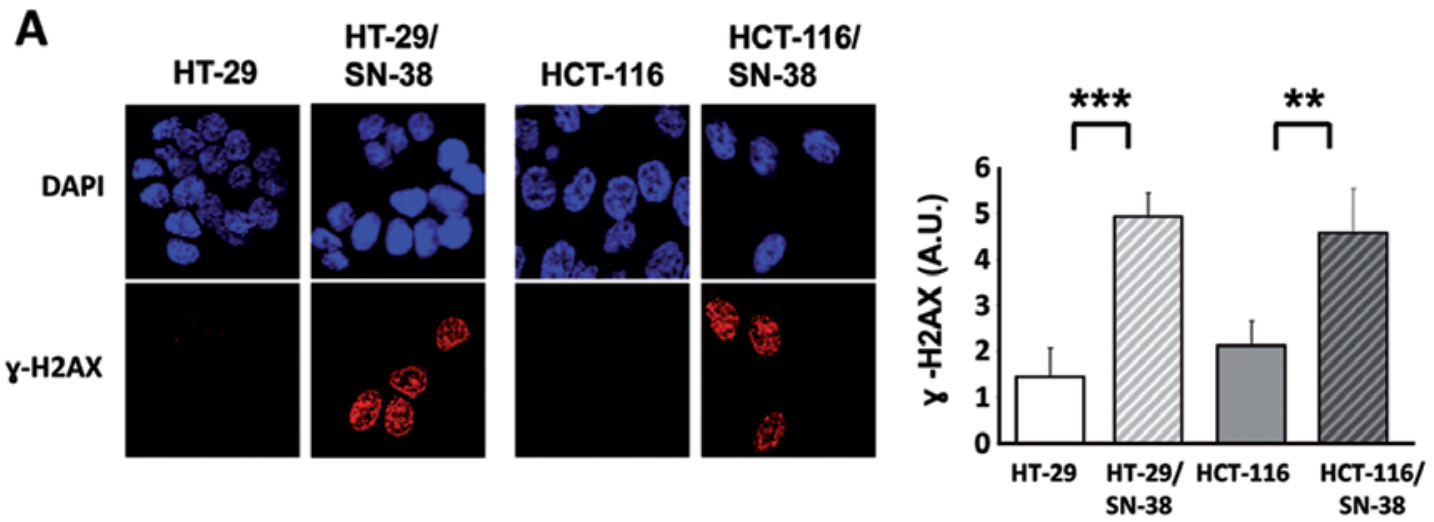

B

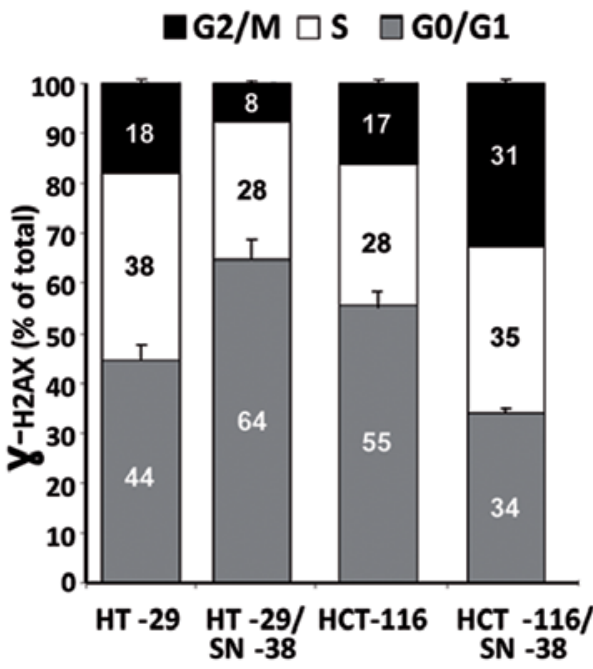

C

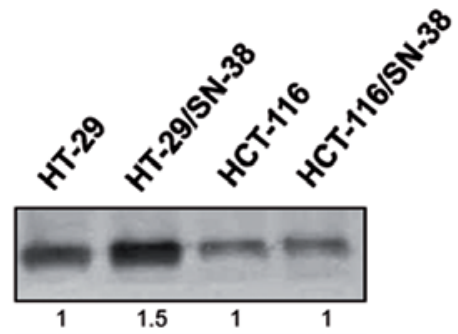

Chk-1

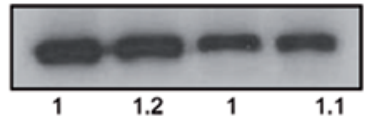

p-Chk-2

(Thr 68)

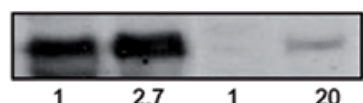

Chk-2

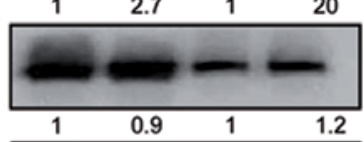

$\beta$-actin

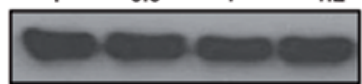

Figure 4. Expression of phosphorylated H2AX, Chk1 and Chk2 in parental and SN-38-resistant colorectal cancer cells. (A) Left, expression of phosphorylated histone H2AX ( $\gamma$-H2AX) (lower panels) was determined by immunofluorescence analysis of untreated HT-29, HT-29/SN-38, HCT-116 and HCT-116/SN-38 cells. The nuclei were counterstained with DAPI and appear in white (upper panels). Right, quantitative image analysis of the signal intensity of phosphorylated H2AX. The data represent the average fluorescence intensity of five independent experiments, each carried out with 1,000 cells. ${ }^{* * * *} \mathrm{p}<0.001,{ }^{* *} \mathrm{p}<0.01$. (B) The expression of $\gamma-\mathrm{H} 2 \mathrm{AX}$ in each part of the cell cycle was determined by biparametric cell cycle analysis. The numbers indicate the fraction (as indicated in $\%$ of total) of the $\gamma$-H2AX for cells in a given part of the cell cycle and are the average of two independent experiments with triplicate samples. Bars, standard deviation. (C) Expression of total and phospho-Chk1 and total and phospho-Chk2 in parental and SN-38-resistant colon cancer cells as determined by western blot analysis with $\beta$-actin as loading control. The numbers represent the relative expression in comparison with the actin control. The numbers indicate the relative expression of the total and phosphorylated forms of Chk1 and Chk2 after standardization for equal loading.

SN-38-resistant cells show increased levels of phosphorylated $H 2 A X$ and Chk2. Phosphorylation of histone H2AX $(\gamma-\mathrm{H} 2 \mathrm{AX})$ is an early response to the formation of DNA double-strand breaks and has also been associated with increased levels of 'oncogenic stress', likely due to replicative errors $(47,48)$. Immunocytochemistry of parental and SN-38-resistant cells showed a significant increase in the basal levels of $\gamma-\mathrm{H} 2 \mathrm{AX}$ for both SN-38-resistant cell lines (Fig. 4A) suggesting that the development of CPT resistance is accompanied by increased activation of the DNA damage response. Biparametric flow cytometry analysis was used to determine whether $\gamma$-H2AX is preferentially associated with a specific phase of the cell cycle (Fig. 4B). The results show that for HT-29 cells, SN-38 resistance is accompanied by an increased fraction of $\gamma-\mathrm{H} 2 \mathrm{AX}$ positive cells in the G1 phase of the cell cycle (from 44 to $64 \%$ ) whereas the fraction of $\gamma-\mathrm{H} 2 \mathrm{AX}$ positive cells in both $\mathrm{S}$ and
$\mathrm{G} 2 / \mathrm{M}$ is decreased. In contrast, for HCT-116 cells, SN-38 resistance is accompanied by an increase of $\gamma$-H2AX positive cells in both the $S$ and G2/M phase of the cell cycle (Fig. 4B). Although apparently contradictory, these results are coherent with the genotype of the investigated cell lines, since MSI cells like HCT-116 are typically diploid due to an efficient mitotic checkpoint that prevents cells with damaged DNA from undergoing mitosis. In comparison, the chromosome abnormalities of CIN cells like HT-29 are, at least in part, due to defects in the mitotic checkpoint allowing mitotic slippage of cells with damaged DNA (11).

The major regulators of the DNA-damage response are the two PIKKs [PI3K (phosphoinoitide 3-kinase)-related kinases], ATM [A-T (ataxia-telangiectasia) mutated] and ATR (ATM- and Rad3-related). ATM and ATR have common phosphorylation substrates such as $\mathrm{H} 2 \mathrm{AX}$, but also distinct 


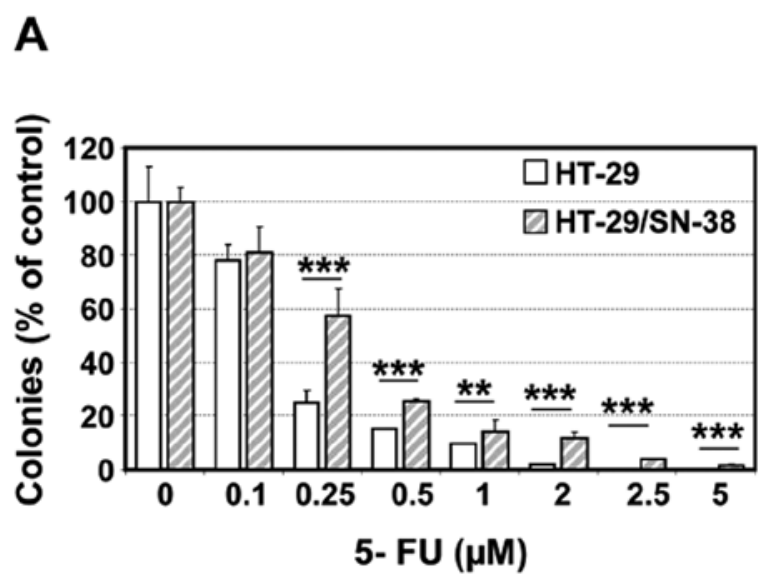

B

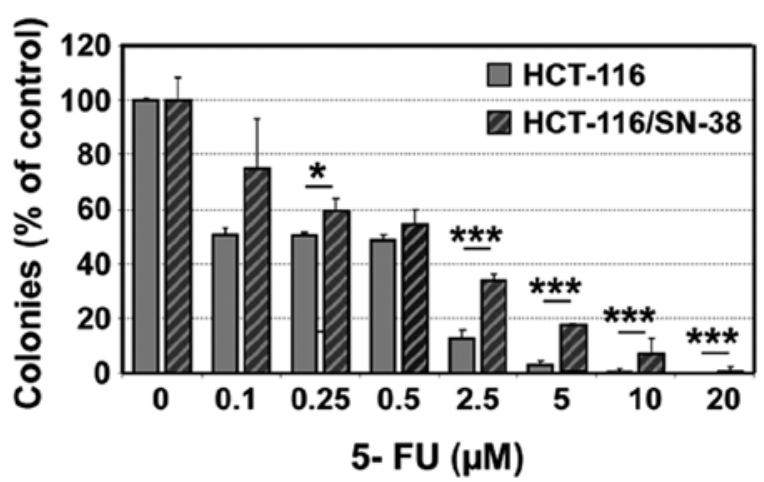

Figure 5. Cytotoxic activity of 5-FU toward parental and SN-38-resistant colorectal cancer cells as determined by the colony formation assay. (A) HT-29 (white columns) and HT-29/SN-38 (hatched columns) cells. (B) HCT-116 (grey columns) and HCT-116/SN-38 (hatched columns) cells. All values represent average values of at least three independent experiments. Bars, standard variation. ${ }^{* * *} \mathrm{p}<0.001,{ }^{* *} \mathrm{p}<0.01,{ }^{*} \mathrm{p}<0.05$

substrates such as the checkpoint kinase Chk2 for ATM and Chk1 for ATR $(36,49,50)$. Western blot analysis of the total and phosphorylated forms of Chk1 showed no consistent differences between parental and SN-38-resistant cells (Fig. 4C). In contrast, phospho-Chk2 was increased in both SN-38 resistant cell lines without changes in Chk2 protein levels. Although the selective activation of Chk2 suggests the involvement of the DNA damage sensor ATM, we found no detectable evidence for increased ATM activation in the SN-38-resistant cells, as indicated by the presence of phospho-Ser1981 ATM (data not shown). Taken together, these findings demonstrate that prolonged exposure to $\mathrm{SN}-38$ is accompanied by an upregulation of the DNA damage response, which is coherent with the increased fraction of cells in the G2 phase of the cell cycle.

SN-38-resistant cells are cross-resistant to 5-fluorouracil. The reduced S-phase fraction suggests that the SN-38-resistant cells might show cross-resistance to $\mathrm{S}$ phase selective anticancer agents. This is particularly relevant for 5-fluorouracil which is frequently administered in combination with irinotecan. Both SN-38 resistant cell lines showed cross-resistance to 5-fluorouracil as determined by colony formation, which was particularly pronounced at high drug concentrations (Fig. 5).
We next compared the sensitivities of HCT-116 and HCT-116/SN-38 xenografts to 5-fluorouracil (25 mg/kg i.p. on days 1, 2, 15 and 16). Treatment with 5-fluorouracil was accompanied by a $75 \%$ decrease in the average tumor volume of HCT-116 tumors compared with a $24 \%$ decrease for HCT-116/SN-38 tumors by day 28 (data not shown). Therefore, acquired resistance to $\mathrm{SN}-38$ was accompanied by crossresistance to 5-FU both in vitro and in vivo.

\section{Discussion}

Irinotecan is approved for treatment of patients with metastatic colorectal cancer $(8,9)$ and shows promising activity in other applications such as gastric cancer. However, even in patients that respond well initially, the activity of irinotecan is eventually limited by acquisition of drug resistance. Although irinotecan is known to be S-phase selective, surprisingly little is known about the influence of prolonged irinotecan exposure on the cell cycle dynamics. Microsatellite instability (MSI) is linked to dysfunction of the DNA mismatch repair (MMR) system, which is involved in the correction of base/base mismatches and insertion/deletion loops during the $\mathrm{S}$ phase of the cell cycle (12). Interestingly, it has been reported that irinotecan is selectively active toward MMR-deficient tumors cells in vitro as well as in patients, compared to tumors with structural and numerical chromosome instability (CIN) $(13,14)$.

We now report the development and characterization of two CRC cell lines, HCT-116/SN-38 (MSI) and HT-29/SN-38 (CIN) with acquired resistance to $\mathrm{SN}-38$, the active metabolite of irinotecan. The parental MSI cells were initially more sensitive to SN-38, compared with the parental CIN cells, in vitro as in vivo, in agreement with previous reports $(13,14)$. However, the MSI cells rapidly developed high levels of SN-38 resistance coherent with the hypermutator phenotype of these cells (11). Overall, we did not observe any notable differences between MSI and CIN cells with regard to the biological modifications observed following prolonged SN-38 exposure.

In agreement with the current model for CPT action, SN-38 resistance was accompanied by decreased levels of SN-38-induced DNA topoisomerase I cleavable complexes. This was principally attributed to an important decrease in drug accumulation which likely is associated with overexpression of one or several ABC transporters. HT-29/SN-38 cells overexpressed both ABCB1 (P-glycoprotein/mdr1) and ABCG2 (breast cancer related protein/mitoxantrone transporter/BCRP) whereas neither parental nor resistant HCT-116 cells expressed these two proteins. However, the decreased drug uptake in HCT-116/SN-38 cells might involve members of the MDR/ ABCC family (21).

Strikingly, both SN-38 resistant cell lines displayed permanent modifications of the cell cycle dynamics under basal conditions (that is under drug-free conditions). Specifically, SN-38 resistant cell lines showed a prolonged generation doubling time which was accompanied by a lower proportion of $\mathrm{S}$ phase cells and a doubling of the fraction of cells in G2/M. Subsequent characterization of cells with cytoplasmic cyclin B1, a marker for cells in G2, in combination with quantitative analysis of cells expressing phospho-H3, a mitotic marker, unambiguously showed that the increased G2/M fraction was due to an increase of cells in the G2 phase of the cell cycle. 
The altered growth dynamics was confirmed in vivo for tumor xenografts established from parental and SN-38-resistant cells. In particular, by 28 days the average tumor size of the CPT-resistant xenografts was approximately half of that observed for the corresponding parental tumors. In addition, in vivo DNA synthesis (as determined by EdU incorporation) was significantly reduced for both tumor models.

The altered growth dynamics might influence the sensitivity to other anticancer agents. 5-Fluorouracil is of particular interest since it is an S-phase selective agent which is often administered in combination with irinotecan. Indeed, both SN-38-resistant cell lines showed cross-resistance to 5-fluorouracil as determined by colony formation assays which was subsequently confirmed in vivo for the SN-38 resistant HCT-116 cells.

Increased activation of proteins involved in the DNA damage response, including the phosphorylated forms of histone $\mathrm{H} 2 \mathrm{AX}(\gamma-\mathrm{H} 2 \mathrm{AX})$ and the checkpoint kinases Chk1 and Chk2 has been associated with 'oncogenic stress', probably linked to replication-induced DNA damage. This type of DNA damage response is quantitatively and temporally different from the classical DNA damage response observed after acute genotoxic stress like ionizing radiation. Specifically, replicative stress is associated with a modest, but permanent activation of the DNA damage response compared to the strong but transient response following ionizing irradiation $(47,48)$. We show here that both SN-38-resistant cell lines had increased basal levels of phosphorylated $\mathrm{H} 2 \mathrm{AX}$ and Chk2, but not Chk1. This is in agreement with previous studies reporting that certain types of replicationdependent DNA damage may activate the ATM/Chk2 pathway rather than the canonical S phase-specific ATR/Chk1 checkpoint pathway (51). Although the selective activation of Chk2 points toward the involvement of the DNA damage sensor ATM, we found no evidence for ATM activation, as indicated by the presence of phospho-Ser1981ATM. However, it is possible that the putative ATM activation in the SN-38-resistant cells might be below the detection limit. An alternative explanation is that Chk 2 might have been activated by the DNA-dependent protein kinase, which targets the same residue on Chk2 as ATM (52).

Our data are in line with the increasing experimental and clinical evidence indicating that exposure to cancer therapeutics may not only promote classical resistance mechanisms but also change the biology of the tumor. Identification of these mechanisms represents a major challenge in the years to come considering that such changes may be accompanied either by cross-resistance or by increased sensitivity to other classes of therapeutic agents.

In summary, we present evidence that prolonged exposure to SN-38/irinotecan is accompanied by permanent modifications of cell cycle dynamics in vitro as in vivo. This could have a profound impact on tumor sensitivity to a wide range of structurally unrelated antitumor agents and may influence tumor progression in patients.

\section{Acknowledgements}

This study was supported in part by the Association pour la Recherche sur le Cancer (ARC), Villejuif, France and Institut National du Cancer (INCa, CETIRICOL, PL06.008). A.P. was supported by a fellowship from the Fondation pour la Recherche Médicale (FRM), France. The authors thank Delphine Muller,
Tatiana Ledent and Olivier Bernadini from the animal facilities at Saint-Antoine Research Center (PHEA); Sylvie Dumont and Fatiha Merabtene from the pathology service and Anne-Marie Faussat from the flow cytometry service, both IFR65.

\section{References}

1. Hsiang YH, Lihou MG and Liu LF: Arrest of replication forks by drug-stabilized topoisomerase I-DNA cleavable complexes as a mechanism of cell killing by camptothecin. Cancer Res 18: 5077-5082, 1989.

2. Larsen AK, Gilbert C, Chyzak G, Plisov SY, Naguibneva I, Lavergne O, Lesueur-Ginot L and Bigg DC: Unusual potency of BN 80915, a novel fluorinated E-ring modified camptothecin, toward human colon carcinoma cells. Cancer Res 7: 2961-2967, 2001.

3. Wu J, Yin MB, Hapke G, Tóth K and Rustum YM: Induction of biphasic DNA double-strand breaks and activation of multiple repair protein complexes by DNA topoisomerase I drug 7-ethyl10-hydroxy-camptothecin. Mol Pharmacol 4: 742-748, 2002.

4. Regairaz M, Zhang YW, Fu H, Agama KK, Tata N, Agrawal S, Aladjem MI and Pommier Y: Mus81-mediated DNA cleavage resolves replication forks stalled by topoisomerase I-DNA complexes. J Cell Biol 195: 739-749, 2011.

5. Horwitz SB and Horwitz MS: Effects of camptothecin on the breakage and repair of DNA during the cell cycle. Cancer Res 33: 2834-2836, 1973.

6. Cheng MF, Chatterjee S and Berger NA: Schedule-dependent cytotoxicity of topotecan alone and in combination chemotherapy regimens. Oncol Res 6: 269-279, 1994.

7. Huang $X$, Traganos F and Darzynkiewicz Z: DNA damage induced by DNA topoisomerase I- and topoisomerase II-inhibitors detected by histone $\mathrm{H} 2 \mathrm{AX}$ phosphorylation in relation to the cell cycle phase and apoptosis. Cell Cycle 6: 614-619, 2003.

8. Saltz LB, Cox JV, Blanke C, Rosen LS, Fehrenbacher L, Moore MJ, Maroun JA, Ackland SP, Locker PK, Pirotta N, Elfring GL and Miller LL: Irinotecan plus fluorouracil and leucovorin for metastatic colorectal cancer. Irinotecan Study Group. N Engl J Med 13: 905-914, 2000.

9. Douillard JY, Cunningham D, Roth AD, Navarro M, James RD, Karasek P, Jandik P, Iveson T, Carmichael J, Alakl M, Gruia G, Awad L and Rougier P: Irinotecan combined with fluorouracil compared with fluorouracil alone as first-line treatment for metastatic colorectal cancer: a multicentre randomised trial. Lancet 9209: 1041-1047, 2000.

10. Lengauer C, Kinzler KW and Vogelstein B: Genetic instabilities in human cancers. Nature 6712: 643-649, 1998.

11. Cancer Genome Atlas Network: Comprehensive molecular characterization of human colon and rectal cancer. Nature 487: 330-337, 2012.

12. Jiricny J: The multifaceted mismatch-repair system. Nat Rev Mol Cell Biol 5: 335-346, 2006.

13. Jacob S, Aguado M, Fallik D and Praz F: The role of the DNA mismatch repair system in the cytotoxicity of the topoisomerase inhibitors camptothecin and etoposide to human colorectal cancer cells. Cancer Res 17: 6555-6562, 2001.

14. Fallik D, Borrini F, Boige V, Viguier J, Jacob S, Miquel C, Sabourin JC, Ducreux M and Praz F: Microsatellite instability is a predictive factor of the tumor response to irinotecan in patients with advanced colorectal cancer. Cancer Res 18: 5738-5744, 2003.

15. Kanzawa F, Sugimoto Y, Minato K, Kasahara K, Bungo M, Nakagawa K, Fujiwara Y, Liu LF and Saijo N: Establishment of a camptothecin analogue (CPT-11)-resistant cell line of human non-small cell lung cancer: characterization and mechanism of resistance. Cancer Res 18: 5919-5924, 1990.

16. Maliepaard M, van Gastelen MA, de Jong LA, Pluim D, van Waardenburg RC, Ruevekamp-Helmers MC, Floot BG and Schellens JH: Overexpression of the BCRP/MXR/ABCP gene in a topotecan-selected ovarian tumor cell line. Cancer Res 18: 4559-4563, 1999.

17. Kawabata S, Oka M, Shiozawa K, Tsukamoto K, Nakatomi K, Soda H, Fukuda M, Ikegami Y, Sugahara K, Yamada Y, Kamihira S, Doyle LA, Ross DD and Kohno S: Breast cancer resistance protein directly confers SN-38 resistance of lung cancer cells. Biochem Biophys Res Commun 5: 1216-1223, 2001. 
18. Rasheed ZA and Rubin EH: Mechanisms of resistance to topoisomerase I-targeting drugs. Oncogene 22: 7296-7304, 2003.

19. Boyer J, McLean EG, Aroori S, Wilson P, McCulla A, Carey PD, Longley DB and Johnston PG: Characterization of p53 wild-type and null isogenic colorectal cancer cell lines resistant to 5-fluorouracil, oxaliplatin, and irinotecan. Clin Cancer Res 6: 2158-2167, 2004.

20. Arakawa Y, Suzuki H, Saito S and Yamada H: Novel missense mutation of the DNA topoisomerase I gene in SN-38-resistant DLD-1 cells. Mol Cancer Ther 5: 502-508, 2006.

21. Takara K, Kitada N, Yoshikawa E, Yamamoto K, Horibe S, Sakaeda T, Nishiguchi K, Ohnishi N and Yokoyama T: Molecular changes to HeLa cells on continuous exposure to SN-38, an active metabolite of irinotecan hydrochloride. Cancer Lett 1: 88-96, 2009.

22. Tagen M, Zhuang Y, Zhang F, Harstead KE, Shen J, Schaiquevich P, Fraga CH, Panetta JC, Waters CM and Stewart CF: P-glycoprotein, but not multidrug resistance protein 4, plays a role in the systemic clearance of irinotecan and SN-38 in mice. Drug Metab Lett 4: 195-201, 2010.

23. Pastorino F, Loi M, Sapra P, Becherini P, Cilli M, Emionite L, Ribatti D, Greenberger LM, Horak ID and Ponzoni M: Tumor regression and curability of preclinical neuroblastoma models by PEGylated SN38 (EZN-2208), a novel topoisomerase I inhibitor. Clin Cancer Res 19: 4809-4821, 2010.

24. Waterhouse DN, Yapp D, Verreault M, Anantha M, Sutherland B and Bally MB: Lipid-based nanoformulation of irinotecan: dual mechanism of action allows for combination chemo/angiogenic therapy. Nanomedicine (Lond) 9: 1645-1654, 2011.

25. Petitprez A and Larsen AK: Irinotecan resistance is accompanied by upregulation of EGFR and Src signaling in human cancer models. Curr Pharm Des: Sep 7, 2012 (Epub ahead of print).

26. Poindessous V, Koeppel F, Raymond E, Comisso M, Waters S and Larsen AK: Marked activity of irofulven (MGI-114) toward human carcinoma cells: comparison with cisplatin and ecteinascidin (ET-743). Clin Cancer Res 9: 2817-2825, 2003.

27. Lemke K, Poindessous V, Skladanowski A and Larsen AK: The antitumor triazoloacridone $\mathrm{C}-1305$ is a topoisomerase II poison with unusual properties. Mol Pharmacol 4: 1035-1042, 2004.

28. Poindessous V, Koeppel F, Raymond E, Cvitkovic E, Waters SJ and Larsen AK: Enhanced antitumor activity of irofulven in combination with 5-fluorouracil and cisplatin in human colon and ovarian carcinoma cells. Int J Oncol 23: 1347-1355, 2003.

29. Larsen AK, Paoletti J, Belehradek J Jr and Paoletti C: Uptake, cytofluorescence, and cytotoxicity of oxazolopyridocarbazoles (amino acid-ellipticine conjugates) in murine sarcoma cells Cancer Res 46: 5236-5240, 1986.

30. LéonceS,Kraus-BerthierL,GolsteynRM,David-CordonnierMH, Tardy C, Lansiaux A, Poindessous V, Larsen AK and Pierré A: Generation of replication-dependent double-strand breaks by the novel N2-G-alkylator S23906-1. Cancer Res 14: 7203-7210, 2006.

31. Rocca CJ, Poindessous V, Soares DG, Ouadrani KE, Sarasin A Guérin E, de Gramont A, Henriques JA, Escargueil AE and Larsen AK: The NER proteins XPC and CSB, but not ERCC1, regulate the sensitivity to the novel DNA binder S23906: implications for recognition and repair of antitumor alkylators. Biochem Pharmacol 3: 335-343, 2010.

32. Pencreach E, Guérin E, Nicolet C, Lelong-Rebel I, Voegeli AC, Oudet P, Larsen AK, Gaub MP and Guenot D: Marked activity of irinotecan and rapamycin combination toward colon cancer cells in vivo and in vitro is mediated through cooperative modulation of the mammalian target of rapamycin/hypoxiainducible factor-1alpha axis. Clin Cancer Res 15: 1297-1307, 2009.

33. Guérin E, Raffelsberger W, Pencreach E, Maier A, Neuville A, Schneider A, Bachellier P, Rohr S, Petitprez A, Poch O, Moras D, Oudet P, Larsen AK, Gaub MP and Guenot D: In vivo topoisomerase I inhibition attenuates the expression of hypoxia-inducible factor $1 \alpha$ target genes and decreases tumor angiogenesis. Mol Med 18: 83-94, 2012.

34. Gil-Delgado MA, Bastian G, Guinet F, Spano JP, Taillibert S, Rocher MA, Castaing D, Adam R, Urien S, Bismuth $\mathrm{H}$ and Khayat D: Oxaliplatin plus irinotecan and FU-FOL combination and pharmacokinetic analysis in advanced colorectal cancer patients. Am J Clin Oncol 27: 294-298, 2004
35. Escargueil AE, Poindessous V, Soares DG, Sarasin A, Cook PR and Larsen AK: Influence of irofulven, a transcription-coupled repair-specific antitumor agent, on RNA polymerase activity, stability and dynamics in living mammalian cells. J Cell Sci 121: $1275-1283,2008$.

36. Soares DG, Battistella A, Rocca CJ, Matuo R, Henriques JA, Larsen AK and Escargueil AE: Ataxia telangiectasia mutatedand Rad3-related kinase drives both the early and the late DNA-damage response to the monofunctional antitumour alkylator S23906. Biochem J 1: 63-73, 2011.

37. Skladanowski A, Côme MG, Sabisz M, Escargueil AE and Larsen AK: Down-regulation of DNA topoisomerase II alpha leads to prolonged cell cycle transit in G2 and early M phases and increased survival to microtubule-interacting agents. Mol Pharmacol 68: 625-634, 2005.

38. Skladanowski A, Bozko P, Sabisz M and Larsen AK: Dual inhibition of PI3K/Akt signaling and the DNA damage checkpoint in p53-deficient cells with strong survival signaling: implications for cancer therapy. Cell Cycle 18: 2268-2275, 2007.

39. Soares DG, Escargueil AE, Poindessous V, Sarasin A de Gramont A, Bonatto D, Henriques JA and Larsen AK: Replication and homologous recombination repair regulate DNA double-strand break formation by the antitumor alkylator ecteinascidin 743. Proc Natl Acad Sci USA 32: 13062-13067, 2007.

40. Soares DG, Machado MS, Rocca CJ, Poindessous V, Ouaret D, Sarasin A, Galmarini CM, Henriques JA, Escargueil AE and Larsen AK: Trabectedin and its $C$ subunit modified analogue PM01183 attenuate nucleotide excision repair and show activity toward platinum-resistant cells. Mol Cancer Ther 8: 1481-1489, 2011.

41. Poindessous V, Ouaret D, El Ouadrani K, Battistella A, Mégalophonos VF, Kamsu-Kom N, Petitprez A, Escargueil AE, Boudou $P$, Dumont $S$, Cervera $P$, Fléjou JF, André $T$, Tournigand C, Chibaudel B, de Gramont A and Larsen AK: EGFR- and VEGF(R)-targeted small molecules show synergistic activity in colorectal cancer models refractory to combinations of monoclonal antibodies. Clin Cancer Res 20: 6522-6530, 2011.

42. Godard T, Deslandes E, Sichel F, Poul JM and Gauduchon P: Detection of topoisomerase inhibitor-induced DNA strand breaks and apoptosis by the alkaline comet assay. Mutat Res 1-2: 47-56, 2002.

43. Hans F and Dimitrov S: Histone H3 phosphorylation and cell division. Oncogene 20: 3021-3027, 2001.

44. Pines J and Hunter T: Cyclin-dependent kinases: a new cell cycle motif? Trends Cell Biol 5: 117-121, 1991.

45. Ookata K, Hisanaga S, Okano T, Tachibana K and Kishimoto T: Relocation and distinct subcellular localization of p34cde2cyclin B complex at meiosis reinitiation in starfish oocytes. EMBO J 5: 1763-1772, 1992

46. Escargueil AE, Plisov SY, Skladanowski A, Borgne A, Meijer L, Gorbsky GJ and Larsen AK: Recruitment of cdc2 kinase by DNA topoisomerase II is coupled to chromatin remodeling. FASEB J 12: 2288-2290, 2001.

47. Bartkova J, Horejsí Z, Koed K, Krämer A, Tort F, Zieger K, Guldberg P, Sehested M, Nesland JM, Lukas C, Ørntoft T, Lukas J and Bartek J: DNA damage response as a candidate anti-cancer barrier in early human tumorigenesis. Nature 7035: 864-870, 2005

48. Gorgoulis VG, Vassiliou LV, Karakaidos P, Zacharatos P, Kotsinas A, Liloglou T, Venere M, Ditullio RA Jr, Kastrinakis NG, Levy B, Kletsas D, Yoneta A, Herlyn M, Kittas C and Halazonetis TD: Activation of the DNA damage checkpoint and genomic instability in human precancerous lesions. Nature 434: 907-913, 2005.

49. Jazayeri A, Falck J, Lukas C, Bartek J, Smith GC, Lukas J and Jackson SP: ATM- and cell cycle-dependent regulation of ATR in response to DNA double-strand breaks. Nat Cell Biol 8: 37-45, 2006.

50. Reinhardt HC and Yaffe MB: Kinases that control the cell cycle in response to DNA damage: Chk1, Chk2 and MK2. Curr Opin Cell Biol 21: 245-255, 2009.

51. Soza S, Leva V, Vago R, Ferrari G, Mazzini G, Biamonti G and Montecucco A: DNA ligase I deficiency leads to replication-dependent DNA damage and impacts cell morphology without blocking cell cycle progression. Mol Cell Biol 29: 2032-2041, 2009.

52. Li J and Stern DF: Regulation of CHK2 by DNA-dependent protein kinase. J Biol Chem 280: 12041-12050, 2005. 